\title{
Note on the Role of Symmetry in Scattering from Isospectral Graphs and Drums
}

\author{
R. BAND ${ }^{a, b, *}$, A. SAWICKI ${ }^{a, c}$ AND U. SMIlANSKY ${ }^{b, d}$ \\ ${ }^{a}$ School of Mathematics, University of Bristol, University Walk, Bristol BS8 1TW, UK \\ ${ }^{b}$ Department of Physics of Complex Systems, the Weizmann Institute of Science, Rehovot 76100, Israel \\ ${ }^{c}$ Center for Theoretical Physics, Polish Academy of Sciences, al. Lotników 32/46, 02-668 Warszawa, Poland \\ ${ }^{d}$ Cardiff School of Mathematics and WIMCS, Cardiff University, Senghennydd Road, Cardiff CF24 4AG, UK \\ We discuss scattering from pairs of isospectral quantum graphs constructed using the method described in \\ the papers of Parzanchevski, Band and Ben-Shach. It was shown in the paper of Band et al. that scattering \\ matrices of such graphs have the same spectrum and polar structure, provided that infinite leads are attached \\ in a way which preserves the symmetry of isospectral construction. In the current paper we compare this result \\ with the conjecture put forward by Okada et al. that the pole distribution of scattering matrices in the exterior of \\ isospectral domains in $\mathbb{R}^{2}$ is different.
}

PACS:

\section{Introduction}

The examination of inverse spectral problems was initiated in 1966 by the famous question of Marc Kac "Can one hear the shape of a drum?" [1]. This question concerns the uniqueness of the spectrum of Laplacian on planar domains with the Dirichlet boundary conditions. A substantial result towards answering Kac's question was due to Sunada who presented a theorem that describes a method for constructing isospectral Riemannian manifolds [2]. In 1992, using an extension of Sunada's theorem, Gordon et al. answered Kac's question as it related to drums, presenting the first pair of isospectral two-dimensional planar domains $[3,4]$. At the same time, the investigation of scattering data started. Examples of objects that share the same scattering information were found both for finite area [5, 6] and infinite area Riemann surfaces $[7,8]$. The search for isospectral and isoscattering examples now includes objects ranging from Riemannian manifolds to discrete graphs. The interested reader can find more about it in the reviews [9-11] and the references within.

The inverse spectral problem for quantum graphs has been first analyzed by Gutkin and Smilansky [12]. The authors of [12] proved that a simple graph with incommensurate lengths of the edges can be fully reconstructed either from the spectrum of its Laplacian or from the overall phase of its scattering matrix. In the recent papers [13-15] a construction method of isospectral and isoscattering quantum graphs was presented.

The work presented in this paper was originally motivated by a paper of Okada et al. [16], in which the scattering from the exterior of isospectral domains in $\mathbb{R}^{2}$ is discussed. The authors suggest that, in spite of the fact

\footnotetext{
* corresponding author; e-mail: rami.band@bristol.ac.uk
}

that the two domains are isospectral, when looked from exterior, the corresponding scattering matrices are not isopolar. This proposition is not proved, but is ushered by heuristic arguments based on the "interior-exterior" duality, and augmented by numerical simulations. The simulations are performed on the isospectral domains in $\mathbb{R}^{2}$ which were constructed by Gordon et al. $[3,4]$ and on further examples by Buser et al. [17]. It is natural to test this conjecture for graphs, which, in spite of being quite simple, usually display most of the complex features which characterize the spectra of domains in $\mathbb{R}^{2}$ studied as interior or exterior problems. Following [15] we prove that for every pair of isospectral quantum graphs obtained from the construction presented in $[13,14]$ the scattering matrices have the same polar structure, provided that leads are attached in a way which preserves the symmetry of isospectral construction. We call such graphs isoscattering. We compare this result with the conjecture put forward by Okada et al. and explain that there is no conflict between these two results. The general proofs of most of the statements can be found in [15]. In the current paper we present some examples and emphasize the role of the symmetry in this problem.

\section{Quantum graphs and scattering matrices}

\subsection{Quantum graphs}

Let $\Gamma=(V, E)$ be a finite graph which consists of $|V|$ vertices that are connected by $|E|$ edges. Each edge, $e \in E$, is a one-dimensional segment of finite length $L_{e}$ with a coordinate $x_{e} \in\left[0, L_{e}\right]$ and this makes $\Gamma$ a metric graph. The metric graph becomes quantum, when we supply it with a differential operator. Here we choose our operator to be the free Schrödinger operator and denote it by $\Delta$. This is merely the one-dimensional Laplacian which equals $-\frac{\mathrm{d}^{2}}{\mathrm{~d} x_{e}^{2}}$ on each of the edges $e \in E$. The 
coupling between the edges is introduced by vertex conditions at the vertices. In the following we will only use the Neumann and Dirichlet vertex conditions (see [18, 19] for other possibilities), which are described below. Let $v \in V$, and $E_{v}$ the set of edges incident to $v$. A function $f$ on $\Gamma$ obeys the Neumann vertex conditions at $v$ if and only if

1. $f$ is continuous at $v$, i.e.,

$$
\forall e_{1}, e_{2} \in E_{v}: f_{e_{1}}(v)=f_{e_{2}}(v) \text {. }
$$

2. The sum of derivatives of $f$ at the vertex $v$ equals zero.

$$
\sum_{e \in E_{v}} \frac{\mathrm{d} f}{\mathrm{~d} x_{e}}(v)=0 .
$$

A function $f$ on $\Gamma$ obeys the Dirichlet vertex conditions at $v$ if and only if

$$
\forall e \in E_{v}: f_{e}(v)=0,
$$

and there are no further requirements on the derivatives.

\subsection{The scattering matrix of a quantum graph}

In this section we explain how to define the scattering matrix of a quantum graph. In order to speak about the scattering problem for a quantum graph we need to connect its vertices (all or a subset) by leads which extend to infinity. For simplicity we assume that leads are connected to vertices of valency greater or equal than two supplied with the Neumann vertex conditions. Let us denote by $\tilde{\Gamma}$ the extended quantum graph which consists of the original graph $\Gamma$ and the external leads $\mathcal{L}$ connected to $M \leq|V|$ vertices which we call the marked vertices. We will not elaborate here on the vertex conditions for $\tilde{\Gamma}$ (see [20] for a more detailed discussion). In the following we will use the two rules:

- the non-marked vertices are supplied with the same vertex conditions as they had in $\Gamma$;

- at each marked vertex $v$ we have Neumann vertex conditions.

We now introduce the scattering matrix which corresponds to $\tilde{\Gamma}$ and denote it by $S_{\tilde{\Gamma}}$.

Let $f$ be an eigenfunction of $\Delta$ with eigenvalue $k^{2}$ and let $\mathcal{L}$ be the set of leads connected to $\Gamma$. The restriction of $f$ to the lead $l \in \mathcal{L}$ can be written in the form

$$
f_{l}\left(x_{l}\right)=a_{l}^{\text {in }} \exp \left(-\mathrm{i} k x_{l}\right)+a_{l}^{\text {out }} \exp \left(\mathrm{i} k x_{l}\right) .
$$

Collecting all the variables $\left\{a_{l}^{\text {in }}\right\}_{l \in \mathcal{L}}$ and $\left\{a_{l}^{\text {out }}\right\}_{l \in \mathcal{L}}$ into vectors which we denote by $\boldsymbol{a}^{\text {in }}$ and $\boldsymbol{a}^{\text {out }}$, we introduce the short-hand notation

$$
\left.f\right|_{\mathcal{L}}=\boldsymbol{a}^{\text {in }} \exp (-\mathrm{i} k x)+\boldsymbol{a}^{\text {out }} \exp (\mathrm{i} k x) .
$$

Using the requirements dictated by the vertex conditions on all the vertices of the graph $\Gamma$, we may write a set of linear equations, some of whose variables are $\left\{a_{l}^{\text {in }}\right\}_{l \in \mathcal{L}}$ and $\left\{a_{l}^{\text {out }}\right\}_{l \in \mathcal{L} \text {. Solving these equations yields relation }}$

$$
\boldsymbol{a}^{\text {out }}=S_{\tilde{\Gamma}}(k) \boldsymbol{a}^{\text {in }} .
$$

The matrix $S_{\tilde{\Gamma}}(k)$ is a square matrix of dimension $|\mathcal{L}|$ and is unitary for every $k \in \mathbb{R}$. This is the scattering matrix of the graph $\tilde{\Gamma}$ (also called the scattering matrix of $\Gamma$ ). The existence and uniqueness of $S_{\tilde{\Gamma}}(k)$ for every value of $k$ and the unitarity of it on the real axis are proved in $[20]$.

\section{Isospectral graphs}

A new construction method of isospectral objects has been recently presented in $[13,14]$. It is a generalization of the well-known Suanda construction [5]. This method can be applied to any geometric object. However here we bring the relevant aspects of the theory as it applies to quantum graphs. In order to avoid quite abstract formalism of representation theory we present the underlying idea on the one particular example (the full discussion of this example, as well as many others can be found in [13]).

\subsection{An example}

Let us consider the graph $\Gamma$ given in Fig. 1a, where $a, b, c$ are lengths of the edges and the vertex conditions at all vertices are Neumann. All the symmetries of $\Gamma$ form a group which is the dihedral group $G=D_{4}$ and it is group of the symmetries of the square. Let us examine two subgroups of $G$ :

$$
H_{1}=\left\{e, r_{u}, r_{v}, \sigma^{2}\right\}, \quad H_{2}=\left\{e, r_{x}, r_{y}, \sigma^{2}\right\},
$$

where $r_{x}, r_{y}, r_{u}, r_{v}$ denote reflections by the axes $x$, $y, u, v$ and $\sigma$ is the counterclockwise rotation by $\frac{\pi}{2}$. Let us consider the following one-dimensional representations $R_{1}$ and $R_{2}$ of $H_{1}$ and $H_{2}$, respectively,

$$
\begin{aligned}
& R_{1}=\left\{e \rightarrow(1), \sigma \rightarrow(-1), r_{v} \rightarrow(-1), r_{u} \rightarrow(1)\right\}, \\
& R_{2}=\left\{e \rightarrow(1), \sigma \rightarrow(-1), r_{y} \rightarrow(1), r_{x} \rightarrow(-1)\right\} .
\end{aligned}
$$

Using these representations we will construct two graphs denoted by $\frac{\Gamma}{R_{1}}$ and $\frac{\Gamma}{R_{2}}$ (Fig. 1b, c) which are isospectral. Now we will explain the process of building the quotient graph $\frac{\Gamma}{R_{2}}$. To this end let us assume that $f$ is the eigenfunction of the Schrödinger operator on the graph $\Gamma$ with eigenvalue $k^{2} \in \mathbb{R}$ which transforms according to the representation $R_{2}$, i.e.

$$
\forall g \in H_{2}: g f=R_{2}(g) f,
$$

where the action of $G$ on $f$ is

$$
[g f](x)=f\left(g^{-1} x\right),
$$

and $R_{2}(g)$ is specified in (5). Since the function $f$ transforms according to the representation $R_{2}$ we know that $r_{x} f=-f$. This implies that $f$ is antisymmetric with respect to the horizontal reflection and in particular vanishes on the fixed points of $r_{x}$. Similarly $r_{y} f=f$ which means that $f$ is symmetric with respect to the vertical reflection, hence the derivative of $f$ vanishes at the fixed 
points of $r_{y}$. Let us notice now that it is enough to know $f$ on the graph shown in Fig. 1c in order to deduce $f$ on the whole graph. The graph shown in Fig. 1c is called the quotient graph $\Gamma / R_{2}$. Repeating the same procedure for $R_{1}$ we obtain the graph $\Gamma / R_{1}$ (see Fig. 1b).
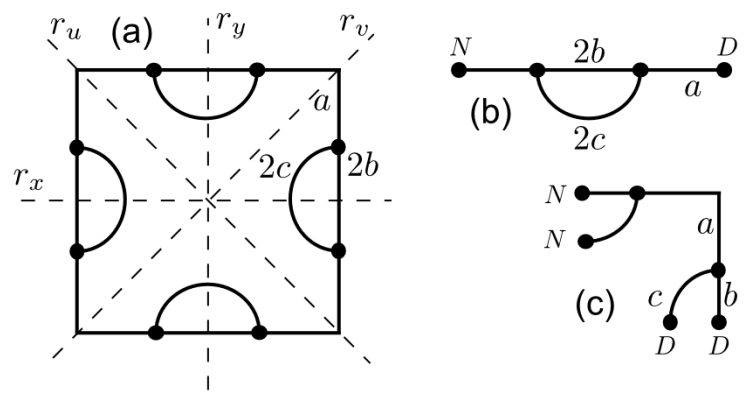

Fig. 1. (a) The graph $\Gamma$ that obeys the dihedral symmetry of the square. The lengths of some edges and the axes of the reflection elements in $D_{4}$ are marked; (b) the graph $\Gamma / R_{1} ;$ (c) the graph $\Gamma / R_{2}$.

It turns out that graphs $\Gamma / R_{1}$ and $\Gamma / R_{2}$ are isospectral $[13,14]$. Moreover the isospectrality of these graphs is due to the fact that

$$
\operatorname{Ind}_{H_{1}}^{G} R_{1} \simeq \operatorname{Ind}_{H_{2}}^{G} R_{2},
$$

and the construction method described above.

\subsection{A transplantation}

Let us denote by $\Phi_{\Gamma}(k)$ the eigenspace of $\Delta$ corresponding to eigenvalue $k^{2}$. In this section we will explain the concept of transplantation. The transplantation is a map between isospectral graphs

$$
T: \Phi_{\Gamma_{1}}(k) \stackrel{\cong}{\longrightarrow} \Phi_{\Gamma_{2}}(k),
$$

which assigns to every eigenfunction on $\Gamma_{1}$ with eigenvalue $k^{2}$ an eigenfunction on $\Gamma_{2}$ with the same eigenvalue $k^{2}$. The way transplantation acts can be easily understood. Let us notice that from Fig. 1b, c we see that the isospectral objects consist of some elementary "building blocks" that are attached to each other in two different prescribed ways. The transplantation can be usually expressed in terms of these building blocks. It expresses the restriction of an eigenfunction to a building block of the first object as a linear combination of the restrictions of an eigenfunction on building blocks of the second object. In case of the graphs in Fig. 1a, b each of them consists of two building blocks and the transplantation matrix from $\Gamma / R_{1}$ to $\Gamma / R_{2}$ is given by

$$
T=\left(\begin{array}{cc}
1 & 1 \\
1 & -1
\end{array}\right) .
$$

It is worth to mention that the existence of transplantation in this case is not a surprise, i.e., the isospectral construction method described in [13, 14] always yields a transplantation.

\section{Isoscattering graphs and drums}

\subsection{Isoscattering graphs}

In this section we give an example of isoscattering graphs, i.e., graphs for which scattering matrices have the same poles structure. The full discussion of a recently presented construction method of isoscattering graphs can be found in [15].

In Sect. 3.1 we explained how to construct isospectral graphs. The method was mainly due to the symmetry of the parent graph $\Gamma$. It is easy to see that we can repeat this procedure for a graph $\tilde{\Gamma}$ with leads (see Fig. 2).
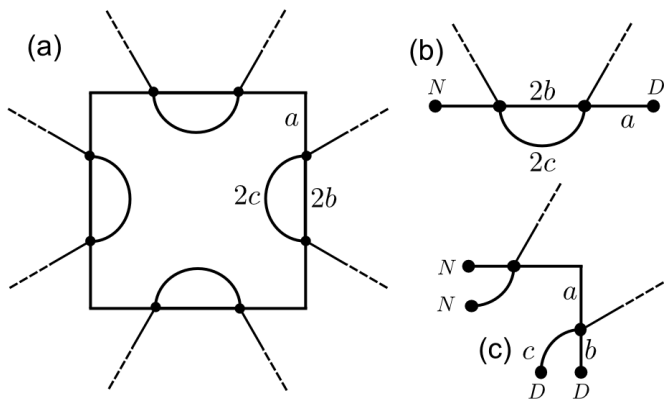

Fig. 2. (a) The graph $\tilde{\Gamma}$ with leads attached; (b) the graph $\tilde{\Gamma} / R_{1} ;$ (c) the graph $\tilde{\Gamma} / R_{2}$.

Moreover, there is a transplantation $T$ between graphs $\tilde{\Gamma} / R_{1}$ and $\tilde{\Gamma} / R_{2}$ and it is given by (10). We know from Sect. 3.2 that the transplantation is a linear transformation which sends every eigenfunction from $\tilde{\Gamma} / R_{1}$ to an eigenfunction of $\tilde{\Gamma} / R_{2}$. The following two observations turn out to be of great importance:

1. It is possible to restrict the transplantation to the leads. This is since a function restricted to a lead of $\tilde{\Gamma} / R_{1}$ can be only send to some linear combination of a function restricted to leads of $\tilde{\Gamma} / R_{2}$ and vice versa.

2. For the graphs $\tilde{\Gamma} / R_{i}$ the restriction of an eigenfunction to the leads is of the form

$$
\begin{aligned}
& \left.f_{\tilde{\Gamma} / R_{i}}\right|_{\mathcal{L}}=\boldsymbol{a}_{\tilde{\Gamma} / R_{i}}^{\mathrm{in}} \exp (-\mathrm{i} k x) \\
& +S_{\tilde{\Gamma} / R_{i}}(k) \boldsymbol{a}_{\tilde{\Gamma} / R_{i}}^{\mathrm{in}} \exp (\mathrm{i} k x),
\end{aligned}
$$

where $S_{\tilde{\Gamma} / R_{i}}(k)$ is the corresponding scattering matrix, $\boldsymbol{a}^{\text {in }} \in \mathbb{C}^{|\mathcal{L}|}$.

The existence of a transplantation together with observation 1 gives

$$
\begin{array}{r}
\left.f_{\tilde{\Gamma} / R_{1}}\right|_{\mathcal{L}}=\boldsymbol{a}_{\tilde{\Gamma} / R_{1}}^{\mathrm{in}} \exp (-\mathrm{i} k x)+S_{\tilde{\Gamma} / R_{1}}(k) \boldsymbol{a}_{\tilde{\Gamma} / R_{1}}^{\mathrm{in}} \exp (\mathrm{i} k x), \\
\downarrow T \\
\left.f_{\tilde{\Gamma} / R_{2}}\right|_{\mathcal{L}}=\boldsymbol{a}_{\tilde{\Gamma} / R_{2}}^{\mathrm{in}} \exp (-\mathrm{i} k x)+S_{\tilde{\Gamma} / R_{2}}(k) \boldsymbol{a}_{\tilde{\Gamma} / R_{2}}^{\mathrm{in}} \exp (\mathrm{i} k x) .
\end{array}
$$


So we obtain

$$
\begin{aligned}
& \boldsymbol{a}_{\tilde{\Gamma} / R_{2}}^{\mathrm{in}}=T \boldsymbol{a}_{\tilde{\Gamma} / R_{1}}^{\mathrm{in}}, \\
& S_{\tilde{\Gamma} / R_{2}}(k) \boldsymbol{a}_{\tilde{\Gamma} / R_{2}}^{\mathrm{in}}=T S_{\tilde{\Gamma} / R_{1}}(k) \boldsymbol{a}_{\tilde{\Gamma} / R_{1}}^{\mathrm{in}} .
\end{aligned}
$$

Finally we get

$$
\begin{aligned}
& S_{\tilde{\Gamma} / R_{2}} T \boldsymbol{a}_{\tilde{\Gamma} / R_{1}}^{\mathrm{in}}=T S_{\tilde{\Gamma} / R_{1}}(k) \boldsymbol{a}_{\tilde{\Gamma} / R_{1}}^{\mathrm{in}} \\
& \quad \Rightarrow T^{-1} S_{\tilde{\Gamma} / R_{2}}(k) T=S_{\tilde{\Gamma} / R_{1}}(k) .
\end{aligned}
$$

The following are now justified:

Theorem 4.1. The scattering matrices of $\tilde{\Gamma} / R_{1}$ and $\tilde{\Gamma} / R_{2}$ are conjugated by the transplantation map for every $k \in \mathbb{C}$.

Corollary 4.1. The scattering matrices of $\tilde{\Gamma} / R_{1}$ and $\tilde{\Gamma} / R_{2}$ have the same polar structure.

This way we get that two graphs $\tilde{\Gamma} / R_{1}$ and $\tilde{\Gamma} / R_{2}$ are isoscattering. It is important to notice that the construction of isoscattering graphs involve the following ingredients/steps:

- A graph $\Gamma$ with certain symmetry group;

- Two isospectral quotient graphs $\Gamma / R_{1}$ and $\Gamma / R_{2}$;

- A graph $\tilde{\Gamma}$ which is an extension of $\Gamma$ by attaching leads to infinity in such a way that the graph $\widetilde{\Gamma}$ obeys the same symmetry group as $\Gamma$;

- Two isoscattering quotient graphs $\tilde{\Gamma} / R_{1}$ and $\tilde{\Gamma} / R_{2}$.

\subsection{Scattering from isospectral drums}

In this section we go back to the conjecture put forward by Okada et al. stating that the pole distribution of scattering matrices in the exterior of isospectral domains in $\mathbb{R}^{2}$ are different. At first glance this conjecture seems to be in contradiction with the result presented in Sect. 4.1. Our main goal is to understand that there is no conflict here.

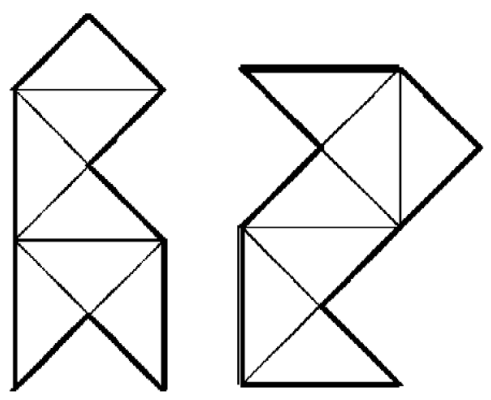

Fig. 3. Two isospectral drums.

Let us first consider the two isospectral drums presented in Fig. 3. Their isospectrality was first proved by Gordon et al. [3]. In Ref. [16] the scattering problem for these two drums was investigated. In particular, the poles structure of scattering matrices of these two drums were computed numerically. The authors of [16] found that these structures are different and hence they concluded that it is possible to distinguish between these two drums while looking from the outside.
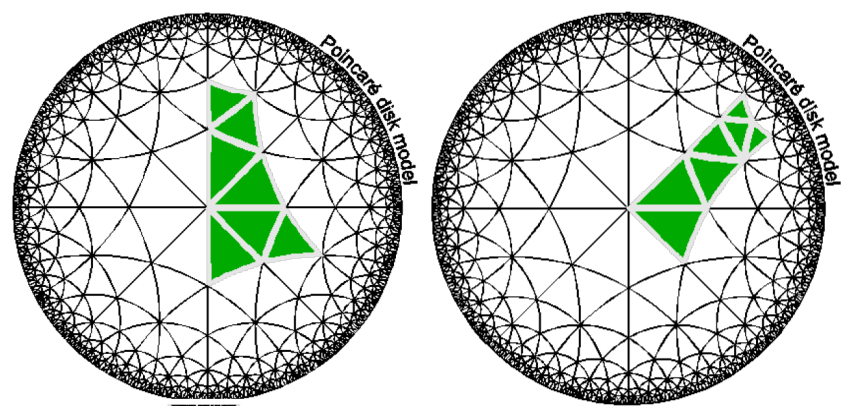

Fig. 4. The hyperbolic plane together with two isospectral hyperbolic drums.

It was first noticed by Buser [17] that the isospectrality of these two drums can be proved using Sunada's construction applied to hyperbolic plane. Sunada's method involves all the elements used in the recently presented method [13, 14], albeit the algebraic condition (8) is restricted to the trivial representations

$$
\operatorname{Ind}_{H_{1}}^{G} \mathrm{id} \simeq \operatorname{Ind}_{H_{2}}^{G} \mathrm{id} .
$$

In particular the construction of quotient is analogous to the one described in Sect. 3.1. We will now describe this construction. To this end we treat the hyperbolic plane (see Fig. 4) as a "graph" with symmetries — each of the lines in Fig. 4 represents one reflection symmetry. Desymmetrization of the hyperbolic plane along some particular reflection lines yields two compact domains denoted in green in Fig. 4. Since our choice of reflection subgroups fulfills the algebraic condition (15) we get that these two domains are isospectral. The immediate consequence of this construction is that the isospectral hyperbolic drums are isometric, hence isoscattering. Since their isospectrality is governed just by the construction method, we can replace the hyperbolic triangles by the Euclidean ones and this way obtain the two isospectral drums shown in Fig. 3. Obviously, the symmetry of the hyperbolic plane is no longer present for these drums. Let us now consider the scattering from the drums shown in Fig. 3. Going back to our graph analogy it is similar to attaching infinite leads to graphs $\Gamma / R_{1}$ and $\Gamma / R_{2}$ in a way which does not come from the original symmetry of $\Gamma$. Then of course the scattering matrices of the corresponding quotient graphs have no longer the same polar structure. In case of drums the same phenomena is present. In order to have the same poles of the scattering matrices we need to consider the scattering problem on the hyperbolic plane. Summing up there is no conflict between our result for quantum graphs and the result of Okada et al. for drums. Moreover the symmetry arguments are responsible for both of them. 


\section{Acknowledgments}

We thank Akira Shudo and Ori Parzanchevski for fruitful discussions. A. Sawicki would like to thank the organizers of 5th Workshop on Quantum Chaos and Localization Phenomena, May 20-22, 2011, Warsaw, Poland for their invitation and hospitality. Grants from EPSRC (grant EP/G021287), ISF (grant 166/09) and BSF (grant 2006065) are acknowledged. The support by Polish MNiSW grant no. N N202 085840 is gratefully acknowledged. R. Band is supported by EPSRC grant no. EP/ $\mathrm{H} 028803 / 1$.

\section{References}

[1] M. Kac, Am. Math. Mon. 73, 1 (1966).

[2] T. Sunada, Ann. Math. 121, 169 (1985).

[3] C. Gordon, D. Webb, S. Wolpert, Bull. Am. Math. Soc. 27, 134 (1992).

[4] C. Gordon, D. Webb, S. Wolpert, Invent. Math. 110, 1 (1992).

[5] P. Berard, Math. Ann. 292, 547 (1992).

[6] Z. Steven, Comm. Partial Diff. Equations 17, 221 (1992).

[7] L. Guillope, M. Zworski, Ann. Math. 145, 597 (1997).
[8] R. Brooks, O. Davidovich, Isoscattering on Surfaces, 2002.

[9] C. Gordon, P. Perry, D. Schueth, Contemp. Math. 387, 157 (2005).

[10] C. Gordon, Contemp. Math. 484, 45 (2009).

[11] R. Brooks, Contemp. Math. 231, 25 (1999).

[12] B. Gutkin, U. Smilansky, J. Phys. A 34, 6061 (2001).

[13] R. Band, O. Parzanchevski, G. Ben-Shach, J. Phys. A, Math. Theor. 42, 175202 (2009).

[14] O. Parzanchevski, R. Band, J. Geometr. Anal. 20, 439 (2010).

[15] R. Band, A. Sawicki, U. Smilansky, J. Phys. A Math. Theor. 43, 415201 (2010).

[16] Y. Okada, A. Shudo, S. Tasaki, T. Harayama, J. Phys. A, Math. Gen. 38, L163 (2005).

[17] P. Buser, J. Conway, P. Doyle, K.-D. Semmler, Int. Math. Res. Not. 9, 391 (1994).

[18] S. Gnutzmann, U. Smilansky, Adv. Phys. 55, 527 (2006).

[19] P. Kuchment, Waves Random Media 14, S107 (2004).

[20] R. Band, G. Berkolaiko, U. Smilansky, Dynamics of nodal points and the nodal count on a family of quantum graphs, in: Ann. Henri Poincaré (2011), http://dx.doi.org/10.1007/s00023-011-0124-1. 